This item was submitted to Loughborough's Research Repository by the author.

Items in Figshare are protected by copyright, with all rights reserved, unless otherwise indicated.

\title{
Coupled level-set volume of fluid simulations of water flowing over a simplified drainage channel with and without air coflow
}

PLEASE CITE THE PUBLISHED VERSION

http://dx.doi.org/10.4271/2017-01-1552

PUBLISHER

(C) SAE International

VERSION

VoR (Version of Record)

\section{PUBLISHER STATEMENT}

This work is made available according to the conditions of the Creative Commons Attribution-NonCommercialNoDerivatives 4.0 International (CC BY-NC-ND 4.0) licence. Full details of this licence are available at: https://creativecommons.org/licenses/by-nc-nd/4.0/

\section{LICENCE}

CC BY-NC-ND 4.0

\section{REPOSITORY RECORD}

Dianat, Mehriar, Maciej Skarysz, Graham Hodgson, Andrew Garmory, and Martin Passmore. 2017. "Coupled Level-set Volume of Fluid Simulations of Water Flowing over a Simplified Drainage Channel with and Without Air Coflow". figshare. https://hdl.handle.net/2134/24494. 


\title{
Coupled Level-Set Volume of Fluid Simulations of Water Flowing Over a Simplified Drainage Channel With and Without Air Coflow
}

\author{
Mehriar Dianat, Maciej Skarysz, Graham Hodgson, Andrew Garmory, and Martin Passmore \\ Loughborough University
}

\begin{abstract}
The motivation for this paper is to predict the flow of water over exterior surfaces of road vehicles. We present simulations of liquid flows on solid surfaces under the influence of gravity with and without the addition of aerodynamic forces on the liquid. This is done using an implementation of a Coupled Level Set Volume of Fluid method (CLSVOF) multiphase approach implemented in the open source OpenFOAM CFD code. This is a high fidelity interface-resolving method that solves for the velocity field in both phases without restrictions on the flow regime. In the current paper the suitability of the approach to Exterior Water Management (EWM) is demonstrated using the representative test cases of a continuous liquid rivulet flowing along an inclined surface with a channel located downstream perpendicular to the oncoming flow. Experimental work has been carried out to record the motion of the rivulet in this case and also to measure the contact angle of the liquid with the solid surface. The measurements of the liquid/solid characteristics such as equilibrium and dynamic contact angles are described along with the analytical expression for contact angle vs. capillary number used in the CFD code. The results from the simulations are compared to experimental measurements. The simulations are carried out with air co-flows of $0,0.5$ and $10 \mathrm{~m} / \mathrm{s}$. The simulations are seen to reproduce physical phenomena such as the liquid pinning at sharp corners and the longitudinal stretching of the rivulet with higher air velocity.
\end{abstract}

CITATION: Dianat, M., Skarysz, M., Hodgson, G., Garmory, A. et al., "Coupled Level-Set Volume of Fluid Simulations of Water Flowing Over a Simplified Drainage Channel With and Without Air Coflow," SAE Int. J. Passeng. Cars - Mech. Syst. 10(1):2017, doi:10.4271/201701-1552.

\section{INTRODUCTION}

Predicting and controlling the motion of rainwater over the exterior of road vehicles, EWM, is an important consideration for vehicle designers. Phenomena such as A-pillar overflow can obscure a driver's vision and droplets or rivulets falling on passengers or their belongings can be a significant source of customer dissatisfaction. There are a wide range of engineering applications involving the flow of liquid films, rivulets and droplets over solid surfaces. For example, water or ice on an airplane wing can significantly interfere with its overall performance $[\underline{1}, \underline{2}]$. Rivulets are also observed in coating flows in the chemical industry [ $\underline{3}]$. In the automotive sector examples of EWM simulations can be found in [4] and [ $\underline{5}]$ that both use Lagrangian particle tracking for the airborne droplets and a simple 2D film model for the surface flow. While the approach to the dispersed phase (airborne) may be satisfactory, the assumption that the surface flow can be modelled using a 2D film has limitations. These 2D film models, details of which can be found in [ $[\underline{6}$ for example, solve transport equations for the film thickness but make no attempt to resolve the 3D shape of the surface water. Using such film methods behaviour such as film-stripping and breakup can only be modelled empirically, with the results being valid within certain flow regimes. More fundamentally the two-way interaction between the aerodynamic drag on the water and the shape of the water feature cannot be predicted using such methods.
The objective here is to develop and demonstrate a computational method that can capture the underlying physics and predict the motion of various liquid flow features on the exterior surface of vehicles. By predicting the resolved 3D shape of the droplet it is hoped that this will be more general than existing thin film models. By resolving water features fully in $3 \mathrm{D}$ it is possible to predict droplet breakup or coalescence without the need for empirical modelling as is the case for film models. The most common numerical method for predicting interface resolved two-phase flows is the Volume of Fluid (VOF) method where a conservative transport equation is solved for the fraction of liquid in each cell. See [7] for a review of the approach. Most commercial codes [ㅇ] have an algebraic VOF solver available (where a typical discretisation method is used to convect the VOF value), often with special numerical techniques for sharpening the interface between phases. Despite these techniques, algebraic VOF simulations suffer from numerical diffusion that causes the interface to lose definition; this reduces the accuracy of the predicted shape that in turn affects terms such as surface tension that rely on accurate predictions of surface curvature. A further problem is the choice of interface VOF value. A value $0<\mathrm{VOF}<1$ must be chosen to define the interface but this choice is essentially arbitrary and the value chosen will affect the visualisation of results. Small liquid flow features such as drops can appear to vanish as the VOF value drops below the threshold due to numerical diffusion. Various improvements to the VOF approach have been 
made using more specialist codes, such as 'geometric VOF' (see [7] for details) where the gradient of the VOF field is used along with its local value to define the position of the interface. A VOF approach is combined with a Lattice-Boltzmann solver in [9] which is able to keep the interface (where $0<\mathrm{VOF}<1$ ) confined to a single layer of cells. However, all VOF methods suffer from the difficulty of finding a surface normal and curvature from what is ideally a binary field. This can reduce accuracy in the evaluation of the effect of surface tension. An alternative is to use Level-Set (LS) methods where a transport equation is solved for a signed distance function from the interface [10]. The zero level-set gives a well-defined and sharp interface, allowing the curvature to be found more easily, but as the transport equation is not conservative mass conservation is not guaranteed and high mesh resolution is required. The method used here is a CLSVOF [11] method where both LS and VOF fields are used together to give a method that combines good interface definition and calculation of curvature with mass conservation.

Surface material plays an important role in liquid transport phenomena and the degree to which a material is hydrophilic or hydrophobic will affect how the water behaves. Here the approach is taken of setting the contact angle, as a function of contact line velocity, as a boundary condition using experimental data. To demonstrate the use of our CLSVOF method with contact angle boundary conditions we apply it here to a laboratory test case representative of EWM applications; the flow of a rivulet towards a channel. This is done firstly with the droplet moving under gravity before the simulations are changed to include an air co-flow velocity. An air velocity of $0.5 \mathrm{~m} / \mathrm{s}$ was found to have little effect but a velocity of $10 \mathrm{~m} / \mathrm{s}$ shows significant differences. The method used is described in the following section. Experimental details of the laboratory case used for comparison as well as contact angle measurements are described in Section 2. Finally, Section 3 provides the results of the simulations and some comparison with the experimental data.

\section{COMPUTATIONAL METHOD}

The method is based on a CLSVOF algorithm for interface tracking [11]. It has been implemented [12] into the general purpose open source solver OpenFOAM [13] using a formulation suitable for general unstructured grids. Details of the CLSVOF method implemented in an in-house code for structured grids with no contact models can be found in [14] , and $[\underline{15}, \underline{16}, \underline{17}, \underline{18}]$. The topology of the two-phase flow is represented by two properties; VOF represents the liquid volume fraction and LS is the shortest distance from the interface, positive within the liquid, negative outside and zero on the interface.

The solution domain is first initialised with the initial VOF and LS conditions. The interface is then reconstructed from LS and VOF fields so that the position of the interface within a computational cell and where it intersects cell faces is found. Interface is represented by Piecewise-Linear Interface Calculation (PLIC) with its normal obtained from the LS field and with its location adjusted to give the same volume fraction obtained from the VOF solution. In this way a defined interface position, consistent with both fields, is found and the fraction of each face occupied by liquid can be found from the intersection of the face and the interface plane. This is used to define an Area of Fluid (AOF) as the area of the face occupied by liquid, rather than using traditional discretisation methods. Advection equations are then solved for both VOF and LS.

$$
\begin{gathered}
\frac{\partial \alpha}{\partial t}=-\frac{1}{V} \sum_{i} A O F_{i} \cdot U_{i} \cdot S_{f, i} \\
\frac{\partial \phi}{\partial t}+\nabla(U \phi)=0
\end{gathered}
$$

Here $V$ is the volume of the cell and summation is over all faces in the cell, $S_{f}$ is the face normal vector and $U$ is the velocity vector. AOF is the area of the face occupied by liquid calculated by the intersection of the face and the interface. The VOF equation is solved using an explicit first order Euler temporal solver. The equation for the LS is solved implicitly using a limited Total Variation Diminishing (TVD) scheme for spatial discretisation. Furthermore, a re-initialisation procedure is employed at every time step to ensure that LS stays a distance function in the vicinity of the interface. This is done following the method of Sussman et al [1]].

The velocity field is then found by solving the momentum and pressure equations using the OpenFOAM pressure-velocity PIMPLE correction procedure. The PIMPLE algorithm is the merged PISOSIMPLE predictor-corrector solver for large time step transient incompressible laminar or turbulent flows. It is based on an iterative procedure for solving equations for velocity and pressure. PISO (Pressure Implicit Split Operator) is a transient solver and SIMPLE (Semi Implicit Method for Pressure Linked Equations) is a steadystate solver for incompressible flows. A single set of momentum equations are solved for both phases. The following surface tension effect in the momentum equations is based on the Continuum Surface Force (CSF) [19] with the curvature term calculated from the LS field.

$$
f_{\sigma}=\sigma \kappa \nabla \alpha
$$

where $\sigma$ is the surface tension coefficient of liquid in gas and $\kappa$ is the mean curvature of the free surface calculated from the following equation

$$
\kappa=-\nabla \cdot \frac{\nabla \phi}{|\nabla \phi|}
$$

The variable time step option available in OpenFOAM [13] was used with the maximum global and interface Courant numbers set to 0.5 .

The local density and viscosity are determined using volume weighted averages found from the VOF field. To implement the contact angle model into the CLSVOF formulation the method already available for a generic contact angle model for the interFoam VOF solver in OpenFOAM is followed. This is done by setting the value of LS and VOF for the wall face of an interface cell such that 
the magnitude of their gradients normal to wall are equal to the cosine of the desired contact angle. A key feature of an interface resolving method such as this is that a single velocity and pressure field is solved in space with no restrictions on the topology of the flow (e.g. film, droplets, rivulets etc.).

\section{Computational Domain and Boundary Conditions}

The test case involves the flow of a continuous liquid rivulet along an inclined solid surface of slope $7^{\circ}$ with a channel of width $12.5 \mathrm{~mm}$ and height $2.5 \mathrm{~mm}$ located downstream perpendicular to the oncoming flow. The computational domain is shown in Figure 1. It consist of liquid/air inlets on the left, outflow on the right, solid walls on the floor, side walls or symmetry in the spanwise z-direction and pressure on the top boundary. The floor upstream of the channel has an axial length of $50 \mathrm{~mm}$. Downstream of the channel it is $22.5 \mathrm{~mm}$. The width in the spanwise direction is $30 \mathrm{~mm}$ and the vertical height is $12.5 \mathrm{~mm}$. A total hexa mesh of just over a million cells has been used. The grid spacing in the proximity of the walls is around 0.1 $\mathrm{mm}$. The mesh is generally non-uniform being refined near the surface, in the regions close to the channel and in the central region in the spanwise direction where liquid rivulet flows. A typical mesh is shown in Figure 1.
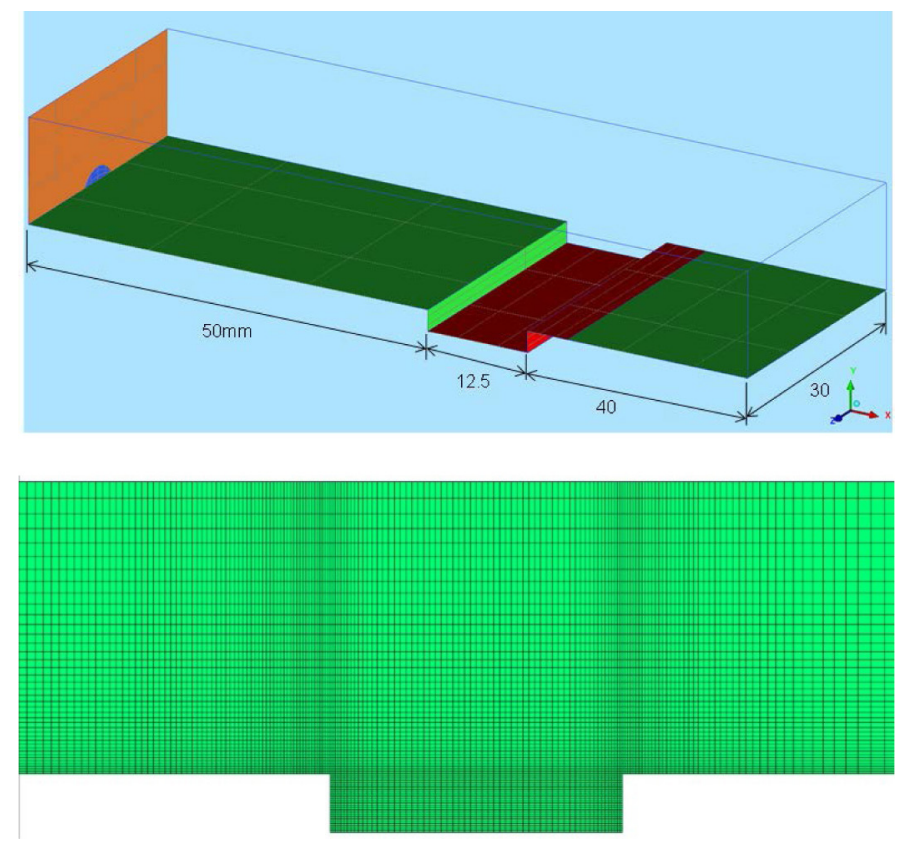

Figure 1. Computational domain and mesh

The liquid is injected through an inlet at the experimentally measured volumetric flow rate of $1 \mathrm{ml} / \mathrm{s}$. At the rivulet front, the measured velocity, once it attains a constant speed, is approximately $U_{L}=16$ $\mathrm{cm} / \mathrm{s}$. Assuming the same velocity at the liquid inlet, the inlet area is then $6.25 \mathrm{~mm}^{2}$. The measured equilibrium contact angle of the upstream surface is $58^{\circ}$. To make the shape of the inlet area consistent with the contact angle, a circular cap is used with the same area and the same measured contact angle at liquid/solid interface. This will lead to a rivulet thickness of around $1.6 \mathrm{~mm}$ and width of around $5.7 \mathrm{~mm}$. The liquid Reynolds number is $\mathrm{Re}_{\mathrm{L}} \sim 909$ and the liquid Weber number is $\mathrm{We}_{\mathrm{L}} \sim 2$ where

$$
R e_{L}=\frac{U_{L} W}{v_{L}}
$$

and

$$
W e_{L}=\frac{\rho_{L} U_{L}^{2} W}{\sigma}
$$

Here $\rho_{L}$ and $v_{L}$ are respectively the liquid density and kinematic viscosity, $W$ is the rivulet width at the inlet and $\sigma=0.072 \mathrm{~N} / \mathrm{m}$ is the surface tension coefficient at water/air interface. The Bond number is around $\mathrm{Bo}=4.4$ where

$$
\text { Bo }=\frac{\rho_{L} g W^{2}}{\sigma}
$$

with $g$ being the gravitational acceleration. Three different conditions were investigated for the air inlet: no air flow, laminar low speed air flow and turbulent higher speed air flow. The former is the test case for which measured data are available while the latter cases were simulated to study the effects of air flow on the rivulet behaviour. With the laminar case, a boundary layer thickness at the inlet was assumed using the Blasius profile

$$
\delta \approx 5 \sqrt{\frac{v x_{o f f}}{U_{0}}}
$$

where $x_{\text {off }}=50 \mathrm{~mm}$ is the offset distance from the start of the boundary layer and $U_{0}=0.5 \mathrm{~m} / \mathrm{s}$ is the free stream velocity. This yields a thickness of around $6 \mathrm{~mm}$ compared to the rivulet thickness of around $1.6 \mathrm{~mm}$. The velocity profile was approximated by a quadratic fit within the boundary layer thickness. With turbulent air flow, using the same offset, a boundary layer thickness was assumed using

$$
\delta \approx \frac{0.37 x_{o f f}}{R e_{x}^{0.2}}
$$

Where

$$
R e_{x}=\frac{U_{0} x_{o f f}}{v}
$$

is the Reynolds number. For this case, the free stream velocity is now $10 \mathrm{~m} / \mathrm{s}$. This would give a thinner boundary layer thickness of around $2.3 \mathrm{~mm}$. For the mean velocity, the $1 / 7$ th power-law profile was fitted. This can be thought of as representative of the early part of a boundary layer on a vehicle moving at moderate road speed. In addition, random perturbations with Gaussian distribution were added to all three components of the velocity with the standard deviation of $10 \%$ of the free stream velocity. Future work will investigate the use of fully correlated synthetic inlet turbulence but this is not available 
in the version of OpenFOAM employed. The solution method used for solving the Navier-Stokes equations is based on Large Eddy Simulation (LES) using the Smagorinsky subgrid-scale model with van Driest damping near the wall. The corresponding gas Reynolds number for the laminar and turbulent flows are respectively $\operatorname{Re}_{\mathrm{G}} \sim$ 189 and 3787 where

$$
\operatorname{Re}_{G}=\frac{U_{G} W}{v_{G}}
$$

with $U_{G}$ the free stream gas velocity and $v_{G}$ the gas kinematic viscosity. The gas Weber numbers are $\mathrm{We}_{\mathrm{G}} \sim 0.024$ and 9.5 where

$$
W e_{G}=\frac{\rho_{G} U_{G}^{2} W}{\sigma}
$$

For both laminar and turbulent cases, the internal velocity field above the floor upstream of the channel was initialised with the boundary layers in the manner described above. For both VOF and LS, the contact angle needs to be imposed as the boundary condition at the liquid/solid interface. The cubic dynamic contact angle model of Cox-Voinov $[\underline{20}, \underline{21}]$ has been used for this purpose. With this model the dynamic contact angle $\theta_{d}$ is obtained from

$$
\theta_{d}^{3}-\theta_{s}^{3}=k . C a
$$

where $k$ is a model parameter given by [22] to be approximately 72 and

$$
\mathrm{Ca}=U_{C L} \mu_{L} / \sigma
$$

is the capillary number. Here, $U_{C L}$ is the contact line velocity and $\mu_{L}$ is the liquid viscosity. The static contact angle, $\theta_{S}$, must be specified for a particular combination of liquid and surface. Values of $\theta_{S}=105^{\circ}$ and $58^{\circ}$ have been used for the channel with hydrophobic plastic material (red colour in Figure 1) and the remaining solid floor with hydrophilic aluminum material (green) respectively as macroscopic contact angles.

For all cases, the VOF and LS fields within the internal cells have been initialised using a spherical cap giving the specified shape at the inlet as described above. The liquid section is initialised with the laminar liquid velocity of $16 \mathrm{~cm} / \mathrm{s}$.

\section{EXPERIMENTAL METHOD}

The experimental element of the work is split into two components. The first relates to obtaining suitable data to allow contact angle simulations, the second is the focus of the study: rivulets. To obtain data for contact angle modelling drops of known volume are placed on a plate at controlled tilt angles. Back-lit images are captured at high resolutions to allow for the identification of the contact angle at different velocities. These data are used in the simulations.
For the rivulet study, a rig was developed to allow production of constant mass-flow rate water rivulets on a plate. The plate was machined aluminum painted to an automotive finish with a channel machined out. Rapid prototype inserts were designed to locate in the channel, permitting a variety of channel geometries to be investigated. A high speed camera was used to obtain footage at 1000 fps with a macro lens attached to obtain a spatial resolution of 13 pixels per mm. Matlab was used to post-process the video footage to obtain the position of the rivulet head in the $x$-direction and the height of fluid at channel entry, mid-channel and channel exit at any frame (Figure 2). These locations were deemed to be critical to the comparison element of the work.

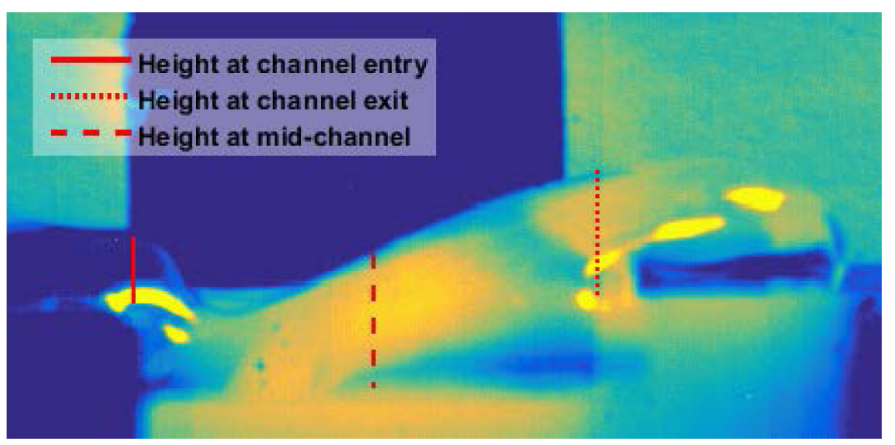

Figure 2. Rivulet dimensions

\section{RESULTS AND DISCUSSION}

\section{Case with No Air Co-Flow}

This section provides comparisons between the experimental and simulation results for the no air flow test case. In Figure 3, numerical predictions of the rivulet front contact line location are compared to the measured data. Here, distance is defined with reference to the channel upstream face location and zero time corresponds to the time when the rivulet front arrives at the channel. Note that due to the way the water overhangs the step as it begins to flow over it, there is a small difference in this region between the contact line and the forward-most point on the liquid. Prior to the channel, a very close agreement with the measured data is observed confirming that the rivulet front velocity once it attains a constant speed is well predicted. This shows the ability of the CLSVOF method to predict correct rivulet behaviour on a flat plate provided a realistic contact angle is specified. The experiment shows that while the leading contact line moves beyond the edge of the downstream edge of the channel it does not continue to flow down the plate. Instead a quasi-equilibrium state is achieved with the water extending $\sim 25 \mathrm{~mm}$ downstream of the channel while the channel fills. This behaviour is not correctly reproduced by the CFD predictions. The simulations do show the water breaching the downstream edge without fully overflowing, most noticeable at $\sim 400 \mathrm{~ms}$ as shown in Figure 4 , but not to the extent seen experimentally. 


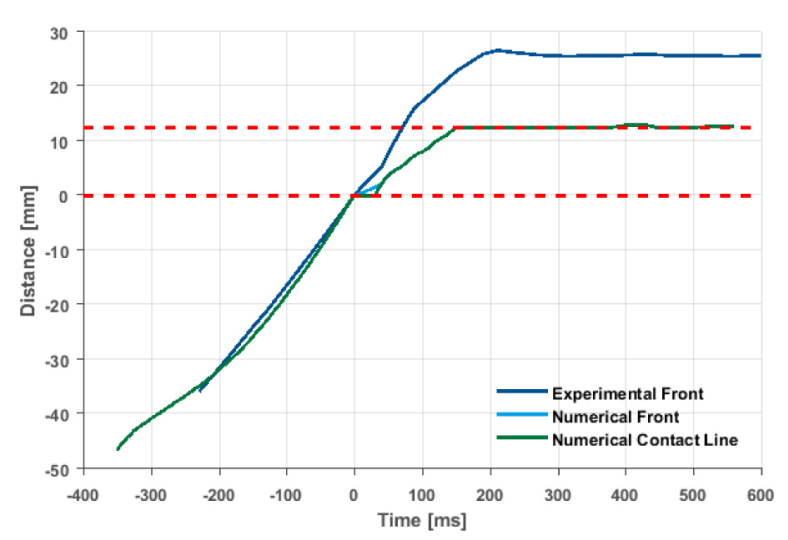

Figure 3. Comparison of simulated and experimental rivulet front location for no air flow test case. Numerical front refers to the furthest most forward point in the liquid at any height while contact line refers to the leading point at the solid surface

Figure 4 shows the simulated liquid position using the $\mathrm{LS}=0$ isosurface coloured by the velocity magnitude that is also used in all subsequent visualisation of the liquid shape in this paper. In VOF methods a choice of threshold VOF value has to be chosen for this type of visualisation and the choice will affect the predicted position. The use of the unambiguous $\mathrm{LS}=0$ isosurface is an advantage of the CLSVOF method.
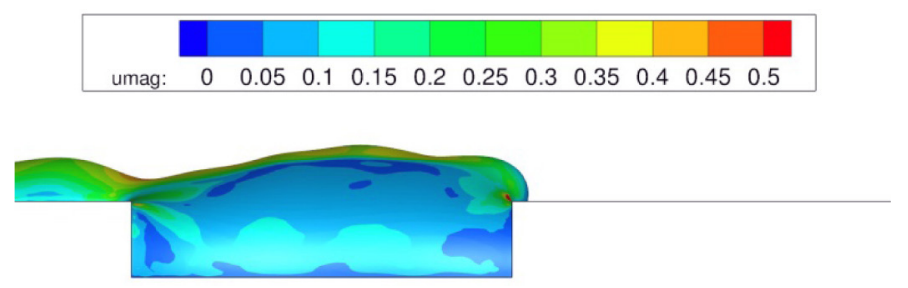

Figure 4. Side view of simulated flow at $\mathrm{t}=430 \mathrm{~ms}$. Note that the water has temporarily overflowed the channel

The discrepancies at the downstream edge can be partly explained by considering the flow at the upstream edge. In Figure 5 images of the experimental rivulet shape near the channel are compared to the simulation results. For both simulations time is relative to when the rivulet contact line reaches the edge of the channel. Once the rivulet front arrives at the channel edge, it pins at that point for a limited duration of order $5 \mathrm{~ms}$. The rivulet front thickens before rolling over and entering into the channel resulting in progressively reduced liquid thickness thereafter. This Gibbs pinning [23] like phenomena is also reproduced in the CFD predictions. Note that during this stage the liquid front location moves ahead of the front contact line even though the floor material in that location is hydrophilic. Such behaviour is more clearly evident in Figure 6 where the predicted rivulet shape in the vicinity of the channel upstream face is shown within a shorter time period. Figure 7 shows a comparison of the predicted and experimental liquid thickness at channel entry. Although the liquid thickness is generally under-predicted, the variation in the shape described above is consistent with the measurements. It is interesting to note that both data and predictions show that the rivulet oscillates during this process presumably due to the interaction of the oncoming flow and the wave propagating upstream during the pinning and liquid thickening. These oscillations are large compared to the pixel size in the experiment and the grid spacing in the simulation and so can safely be concluded not to be an artefact of data processing or visualization. Discrepancies in the liquid thickness at the channel entry are believed largely responsible for the failure of the model to predict the liquid overflowing the channel. As the head of the rivulet in the experiment is larger it will enter the channel with greater momentum that would then carry it across the channel more rapidly and allow it to breach the downstream step to a greater extent than seen in the simulations.
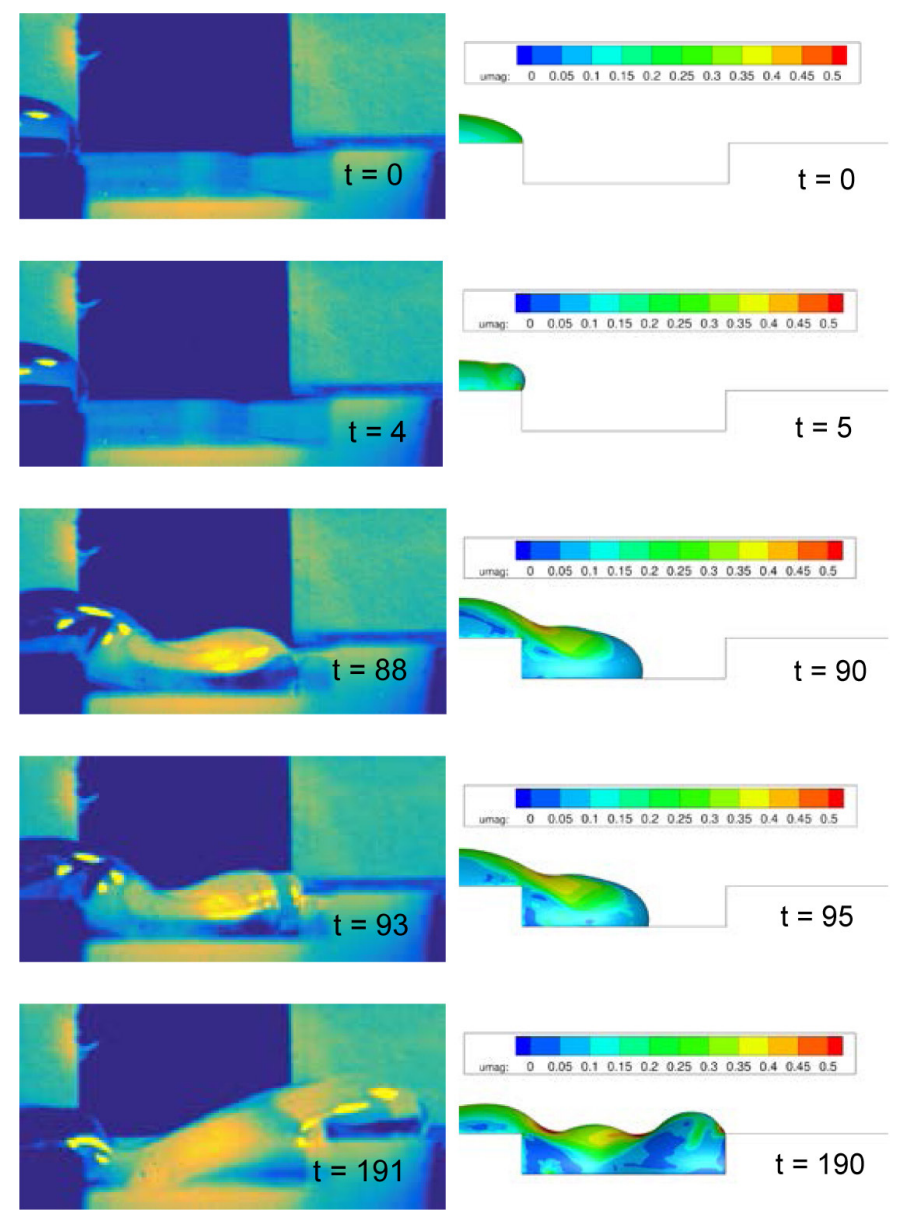

Figure 5. Comparison of rivulet shape. Left: experimental, Right: simulation (time in $\mathrm{ms}$ ). 


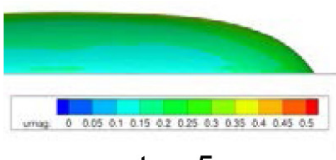

$t=-5$

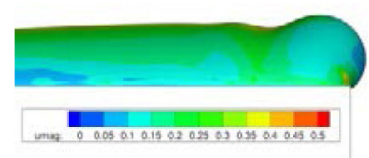

$t=10$

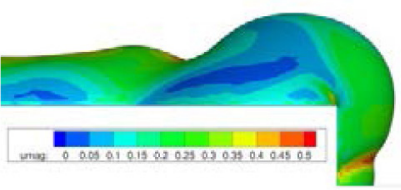

$t=35$
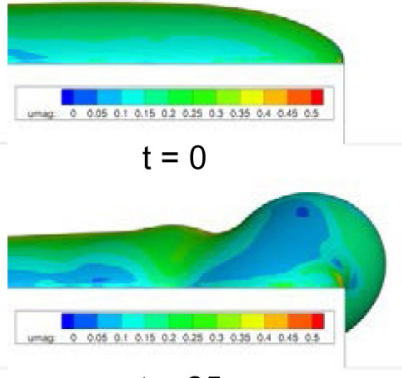

$\mathrm{t}=25$

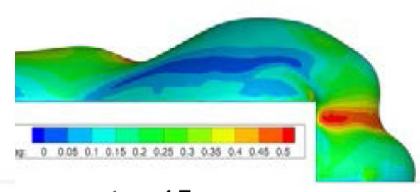

$t=45$
Figure 6. Simulated rivulet shape near the channel entry ( $\mathrm{t}$ in $\mathrm{ms}$ )

Such discrepancies can be caused by a number of factors including uncertainties in experimental measurements. However, the choice of contact angle function is likely to be the cause of much of the discrepancy. The Cox-Voinov type contact angle model was chosen as it requires only the measurement of the static contact angle as well as a single coefficient. Uncertainties in the contact angle were found to significantly influence the rivulet shape, thickness and how far it breaches the channel. Sensitivity studies showed that an increase in equilibrium contact angle from $58^{\circ}$ using the same model resulted in an increase in the liquid thickness as it approaches the channel. When the contact angle for the upstream channel wall was changed to a higher value of $105^{\circ}$, the rivulet was found to overflow the channel due to its increased inertia as it flows into the channel in a manner similar to the experiment. However, this involved changing the static contact angle to values not observed in the experiments and the resulting behaviour is likely to be a poor match with the experiment in other areas such as approach speed. A better method would be to use a more sophisticated relationship between capillary number and contact angle than the one used here. Options include those such as formulated by [24] or [25]. However, these require more detailed measurements of the dynamic contact angle at a range of capillary numbers. This work has indicated the importance of including such data in simulations and ongoing work is focused on carrying out such measurements.

Figure 8 shows examples of the velocity vector field on the mid-plane near the channel edge. A low speed recirculating region is formed within the liquid during the liquid thickening stage just upstream of the channel edge which gradually disappears as the liquid thickness is reduced to its equilibrium level. The gas velocity for this no air flow case is entirely induced by the liquid motion due to gravity. Two-way interaction between the aerodynamic drag and the water flow has been achieved without requiring any empirical models. This figure also stresses the highly 3D character of the flow field and substantiates the earlier remark that the thin liquid film approximation is not suitable for these types of applications.

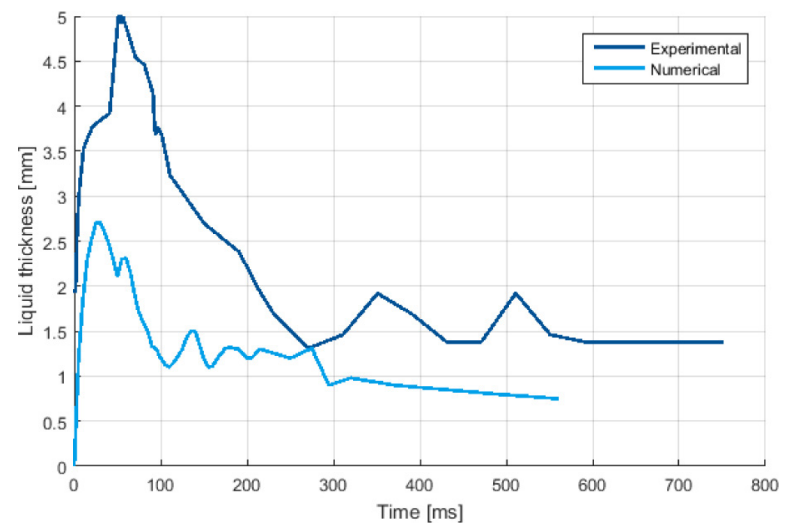

Figure 7. Comparison of simulated and experimental liquid thickness at channel entry for no air flow test case

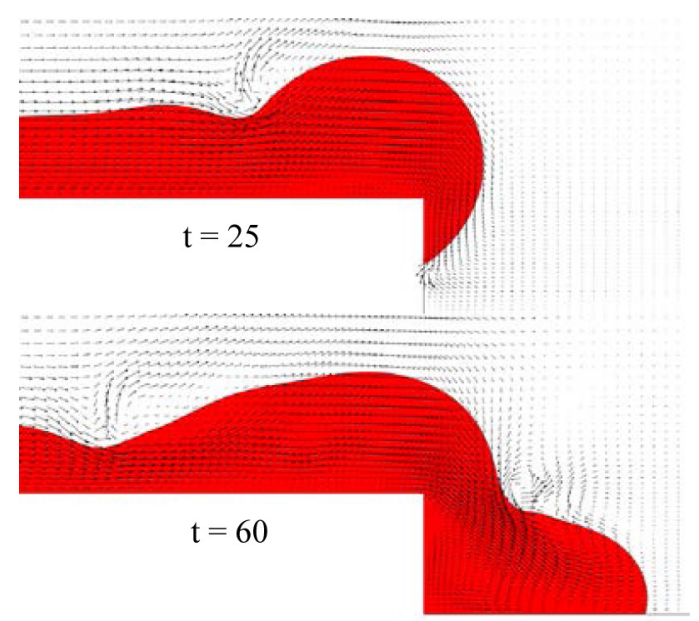

Figure 8. Examples of velocity vector plots near the channel edge

\section{Cases with Air Flow}

To demonstrate the ability of the interface-resolving CFD method to simulate the two-way coupling of aerodynamic drag and liquid topology a non-zero air co-flow at the inlet is studied. In this section the effects caused by the air flow on the rivulet will be described. In Figure 9 the front contact line location is plotted against time for all three cases. The differences between the no air flow and laminar air flow are seen to be small. This is because the momentum is dominated by that of the liquid. However, marked differences exist between the no air flow and the higher speed air flow. With the latter case the rivulet attains a much higher velocity due to the shear force by the air flow. As a consequence it develops a significantly shorter rivulet with narrower width as it is effectively stretched in the axial direction by the air flow. This is confirmed in Figure 10 where the images of the rivulet shape near the channel entry are shown. 


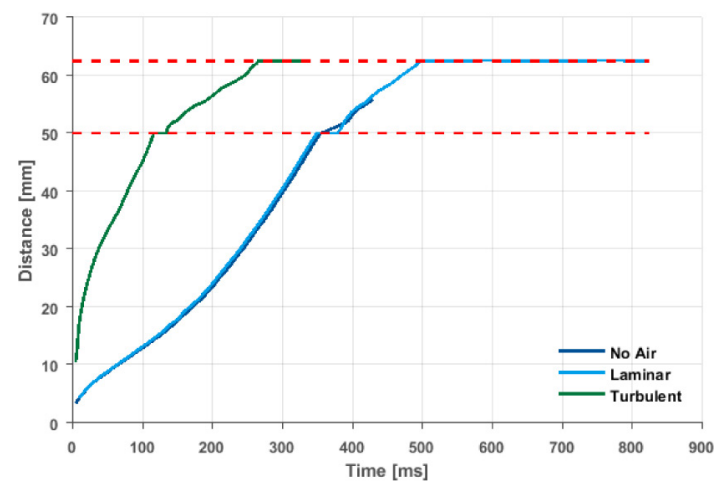

Figure 9. Comparison of simulated rivulet front contact line. Red line indicates channel location. Time is actual time.

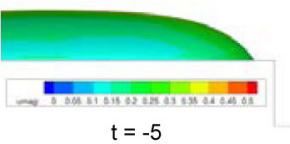

$t=-5$
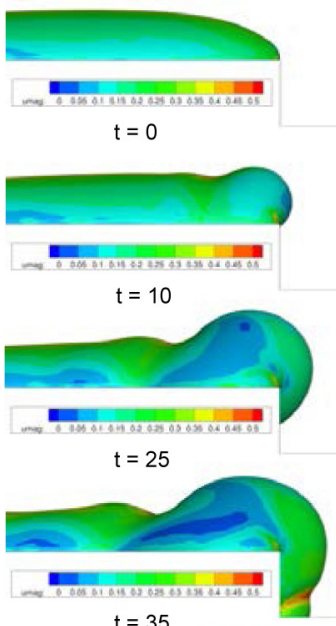

$t=35$

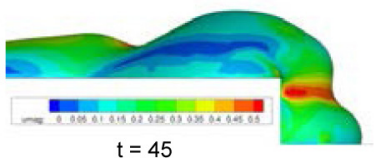

$t=45$
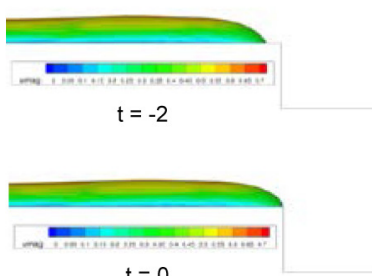

$\mathrm{t}=0$
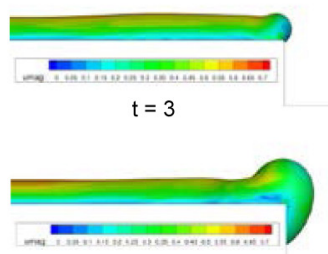

$t=13$
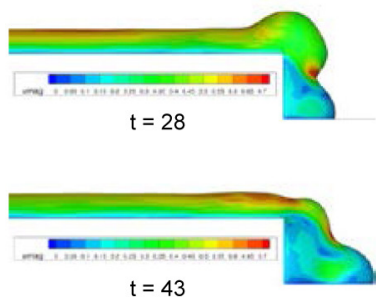

Figure 10. Rivulet shape near channel entry: left: no air flow; right: turbulent air flow ( $\mathrm{t}$ in $\mathrm{ms}$ )

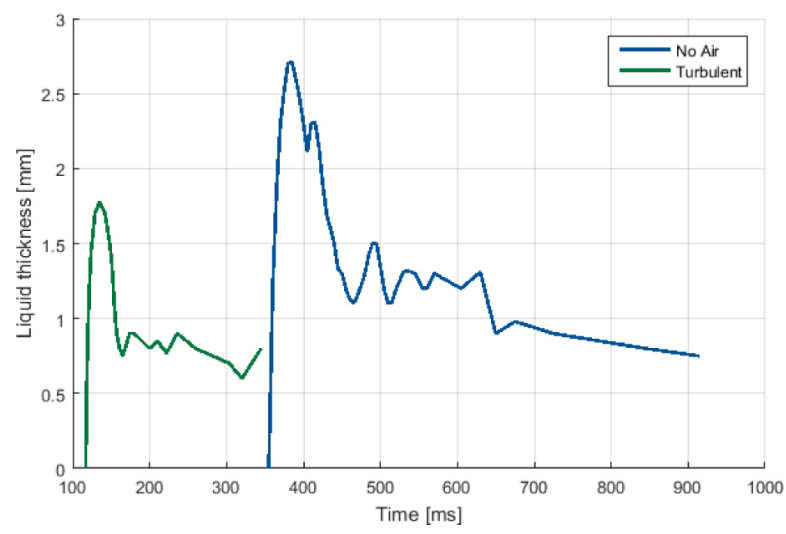

Figure 11. Simulated rivulet height at channel entry. Time is actual time.
In Figure 11 the predicted liquid thickness at channel entry for the no air flow and turbulent air flow cases are compared. The trend is seen to be similar for both cases showing an initial increase followed by the subsequent decrease in rivulet height. In both cases, the rivulet oscillates during this stage as described previously, finally reaching their equilibrium height.

Figure 12 shows an example of the velocity vector plot on the mid-plane near the channel entry for turbulent flow case. Note the significant effect the liquid thickening process has on the gas velocity field. Again as with the no air flow case, two-way coupling between the air and liquid flow is implicit with the current methodology and no empirical models or assumptions are required that are specific to particular test geometries. Also note the formation of a low speed recirculating region upstream of the channel entry during the liquid thickening process. This highly 3D feature of the flow field is further confirmation of the unsuitability of thin liquid film models for such applications.

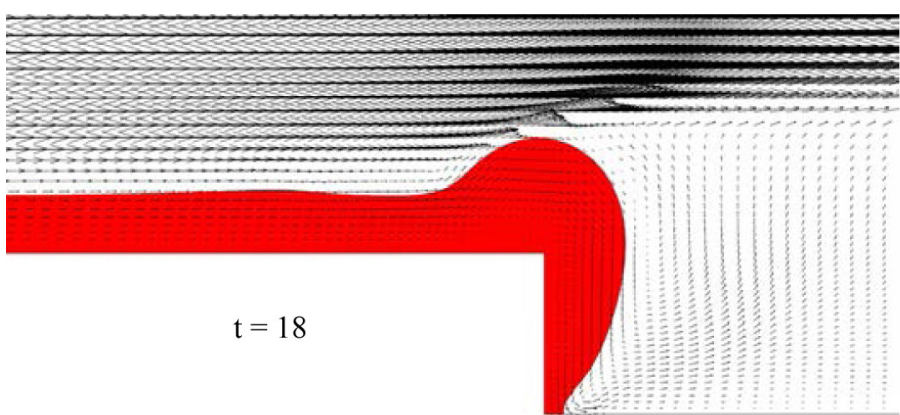

Figure 12. Example of velocity vector plot near the channel edge

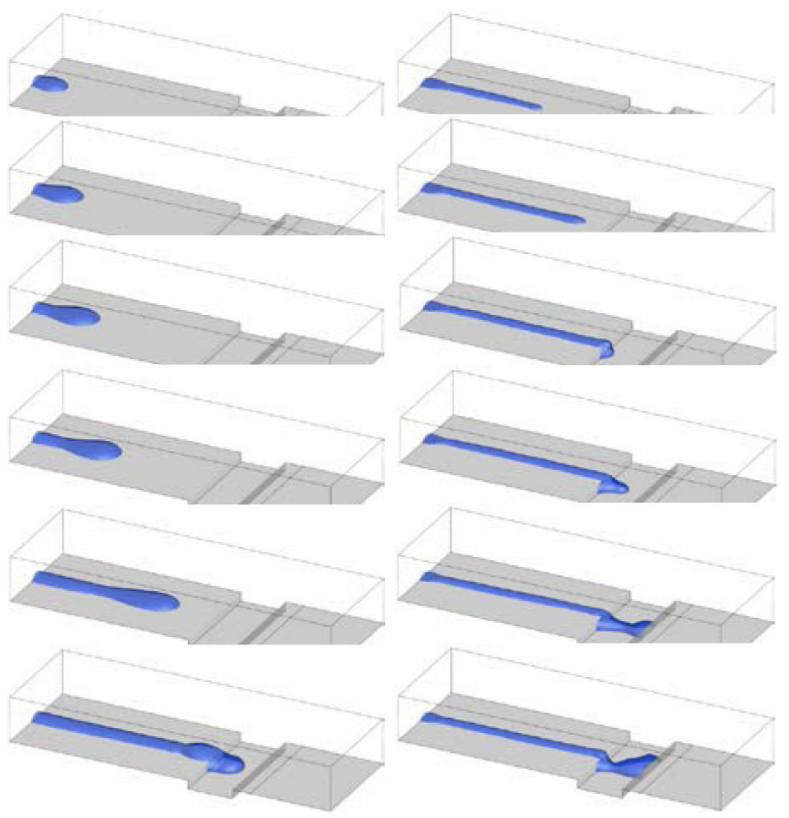

Figure 13. Rivulet shape from top to bottom at $\mathrm{t}=50,100,150,200,300$, $450 \mathrm{~ms}$. Left: no air flow; right: turbulent air flow. Time is the actual simulated time. 
Dianat et al / SAE Int. J. Passeng. Cars - Mech. Syst. / Volume 10, Issue 1 (April 2017)

Figure 13 shows the development of the rivulet shape with time for no air flow and turbulent flow cases. Images are at the same real times to emphasise differences in their shapes, locations, etc. The effect of the rivulet being stretched into a thinner rivulet by the air flow is also seen here. This demonstrates the ability of the method to simulate flows at very different gas Weber numbers. Typically prior knowledge is required of these to select the appropriate model to implement. In both Figure 9 and Figure 13 the different behaviour of rivulet head velocity with time can be seen for the two cases. With no co-flow the rivulet can be seen to accelerate with time due to the effect of gravity, however with a $10 \mathrm{~m} / \mathrm{s}$ co-flow the rivulet is seen to decelerate after an initially higher velocity. This is due to the rivulet deforming to a lower drag shape as it moves, further emphasising the need for interface-resolving methods in EWM simulations.

\section{CONCLUSIONS}

This paper has demonstrated the use of a CLSVOF method with contact angle boundary conditions. CLSVOF combines the advantages of VOF and LS methods to give a method with a well-defined interface location and one that is also mass conservative. This has been applied to the test case of a rivulet approaching a channel as an example of an EWM type application.

The current computational approach adequately predicts the trend in the rivulet behaviour such as its front contact line velocity prior to the entry to the channel and its oscillations and thickening near the channel entry. However, the work has highlighted the need for an improvement in both experiments and computations to enhance the quality of the predictions. The height of the rivulet as it approaches the channel is underpredicted. In turn this means that the rivulet does not enter the channel with sufficient momentum to breach the channel exit to the same extent seen in experiments. The general trend of the rivulet filling the channel rather than escaping downstream is observed; this is difficult to simulate with traditional thin film methods. With the computations, the main discrepancy is likely related to inaccurate specification of the dynamic contact angle for the VOF and LS.

Ongoing work is directed to obtaining experimentally determined contact angle information as a function of capillary number for surfaces used in EWM applications. In addition to accurate measurements of the equilibrium, static receding and static advancing contact angles and dynamic contact angles will also be measured for as wide range of capillary numbers as is practical. Such data will allow the calibration and the use of more suitable dynamic contact angle models that are consistent with the materials used in experiments. Further improvements in the experimental work include improved optical arrangements to obtain increased spatial resolution, overhead imagery and the use of a fluorescing dye to assist in automated post-processing. Future work will also investigate obtaining experimental data for the motion of liquid on surfaces in the presence of an air velocity.
The ability of the method to simulate rivulet flows at a range of gas Weber numbers was also demonstrated by the inclusion of 0.5 and 10 $\mathrm{m} / \mathrm{s}$ air co-flow. The slower air speed was seen to make negligible difference, but significant differences were observed with the higher velocity. The rivulet was seen to move much faster under aerodynamic load and be stretched into a much thinner rivulet. Furthermore, its speed was seen to decrease with distance down the plate due to the full 3D interaction of liquid shape, aerodynamic forces and capillary forces. This highlights one of the advantages of using a fully resolved interface capturing method such as this.

\section{REFERENCES}

1. McAlister, G., Ettema, R. and Marshall, J.S. 2005. Wind-Driven Rivulet Breakoff and Droplet Flows in Microgravity and Terrestrial-Gravity Conditions. ASME J. Fluids Engineering, 127, 257-266

2. Zhang, K. and Hu, H. 2014. An experimental study of the wind-driven water droplet/rivulet flows over an airfoil pertinent to wind turbine icing phenomena. Proc. Of the ASME 2014 4th Joint US-European Fluids Engineering Division Summer Meeting FEDSM2014

3. Marshall, J. S. and Ettema, R. 2005. Contact-line instabilities of driven liquid films. Advances in Fluid Mechanics, WIT Press, Southampton, England

4. Gaylard, A., Fagg, M., Bannister, M., Duncan, B. , "Modelling A-Pillar Water Overflow: Developing CFD and Experimental Methods," SAE Int. J. Passeng. Cars - Mech. Syst. 5(2):789-800, 2012, doi:10.4271/201201-0588

5. Jilesen, J., Gaylard, A., Spruss, I., Kuthada, T., "Advances in Modelling A-Pillar Water Overflow," SAE Technical Paper 2015-01-1549, 2015, doi: $10.4271 / 2015-01-1549$

6. Meredith, K., de Vries, J., Wang, Y. and Xin, Y. 2013. A comprehensive model for simulating the interaction of water with solid surfaces in fire suppression environments. Proceedings of the Combustion Institute, 34, 2719-2726

7. Scardovelli, R. and Zaleski, S. 1999. Direct numerical simulation of free-surface and interfacial flow. Annu. Rev. Fluid Mech.31, 567-603

8. StarCCM+ c 11.02.2016. CD-adapco

9. Korner, C, Thies, M, Hofman, T, Thurey, N and Rude, U. 2005. Lattice Boltzmann Model for Free Surface Flow for Modeling Foaming. J. Stat Phys. 121:179-196

10. Sussman, M., Smereka, P. and Osher, S. 1994. A level set approach for computing solutions to incompressible two-phase flow. J. Comp. Phys., $114,146-159$

11. Sussman, M. and Puckett, E. G. 2000. A coupled level set and volumeof-fluid method for computing $3 \mathrm{~d}$ and axisymmetric incompressible two-phase flows. J. Comp. Phys., 162, 301-337

12. Dianat, M., Skarysz, M. and Garmory, A. 2016. A Coupled Level Set and Volume of Fluid Method for Automotive Exterior Water Management Applications. Submitted to Int. J. Multiphase Flow.

13. OpenFOAM, v.2.1.1, 2013. (http://www.openfoam.com.)

14. Xiao, F. 2012. Large Eddy Simulation of Liquid Jet Primary Breakup. $\mathrm{PhD}$ thesis, Loughborough Univ., U.K

15. Xiao, F., Dianat, M. and McGuirk, J. J. 2013. Large Eddy Simulation of Liquid-Jet Primary Breakup in Air Crossflow. AIAA J., 51, No. 12, 2878-2893

16. Xiao, F., Dianat, M. and McGuirk, J. J. 2014. LES of turbulent liquid jet primary breakup in turbulent coaxial air flow. Int. J. Multiphase Flow, 60, 103-118

17. Xiao, F., Dianat, M. and McGuirk, J. J. 2014. Large Eddy Simulation of Single Drop and Liquid Jet Primary Breakup. Atomization and Sprays, 24, 281-302

18. Xiao, F., Dianat, M. and McGuirk, J. J. 2016. A Robust Interface Method for Drop Formation and Breakup Simulation at High Density Ratio using an Extrapolated Liquid Velocity. Computers and Fluids, 136, 402-420

19. Brackbill, J. U., Kothe, D. B. and Zemach, C. 1992. Continuum Method for Modelling Surface Tension. J. Comp. Phys., 100, 335-354

20. Cox, R. 1998. Inertial and Viscous Effects on Dynamic Contact Angles. J. Fluid Mech., 57, 249-278

21. Voinov, O. 1976. Hydrodynamics of wetting. Fluid Dynamics, 11, 714-721 
22. Hoffman R. L. 1975 A study of the advancing interface. I. Interface shape in liquid-gas systems. J. Colloid Interface Sci., 50, 228

23. Oliver, J. F., Huh, C. and Mason, S. G. 1976. Resistance to Spreading of Liquids by Sharp Edges. Journal of Colloid and Interface Science, 59, 3

24. Shikhmurzaiev Y. D.2008. Capillary Flows with Forming Interfaces. Chapman \& Hall/CRC

25. Yokoi, K., Vadillo, D., Hinch, J. and Hutchings, I. 2009. Numerical studies of the influence of dynamic contact angle on a droplet impacting on a dry surface. Physics of Fluids, 21, 072102

\section{CONTACT INFORMATION}

M. Dianat

m.dianat@lboro.ac.uk

M. Skarysz

m.skarysz@,1boro.ac.uk

G. Hodgson

g.hodgson@lboro.ac.uk

A. Garmory

a.garmory@lboro.ac.uk

M. Passmore

$\underline{\text { m.passmore@lboro.ac.uk }}$

\section{ACKNOWLEDGMENTS}

This work was supported by Jaguar Land Rover and the UK Engineering and Physical Sciences Research Council grant EP/ K014102/1 as part of the jointly funded Programme for Simulation Innovation. Calculations were performed on HPC-Midlands funded by the UK EPSRC, Grant EP/K000063/1. Further thanks to Cameron Millar for the initial experimental data.

\section{DEFINITIONS/ABBREVIATIONS}

\author{
We - Weber Number \\ Re - Reynolds Number \\ Bo - Bond Number \\ Ca - Capillary Number
}

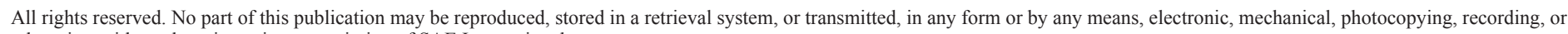
otherwise, without the prior written permission of SAE International. 